\title{
SPATIAL AND TEMPORAL ANALYSIS ON THE EVOLUTION CHARACTERISTICS OF LAND SURFACE TEMPERATURE IN THE PEARL RIVER DELTA REGION
}

\author{
Qiongyao Huang ${ }^{1}$, Juanli Jing ${ }^{1,2^{*}}$ \\ ${ }^{1}$ College of Geomatics and Geo-information, Guilin University of Technology, Guilin, 541004, China \\ ${ }^{2}$ Guangxi Key Laboratory of Spatial Information and Geomatics, Guilin, 541004, China
}

\section{Commission VI, WG VI/4}

KEY WORDS: Pearl River Delta; land surface temperature; spatial and temporal evolution

\section{ABSTRACT:}

With the rapid development of social economy and the continuous progress of urbanization in the Pearl River Delta region, the urban surface temperature had a significant change, which seriously affects people's normal life. The MOD11B3 LST_Day_6km product and other auxiliary data were selected as data sources, and the temperature-standard deviation method, the profile method and the coefficient method were used to analyze the spatial and temporal evolution characteristics of land surface temperature in the Pearl River Delta Region from 2000-2015. The research results showed that:(1) the maximum surface temperature from 2000 to 2015 in June, July and August showed an upward trend, while the minimum temperature showed a fluctuation downward trend, and the average temperature changed gently. (2) the surface temperature had a spatial pattern of low on all sides, and high in the middle. The high temperature zone were mainly distributed in Guangzhou, Dongguan, Foshan and Shenzhen cities and the surrounding areas. And the high temperature areas were more concentrated and showed a spreading direction of East and South. (3) The profile analysis showed that the surface temperature had a double-peak feature along the profile line, and the temperature decreased with the distance farther away from the center of the high temperature zone. (4) The correlation coefficient between land surface temperature and elevation, vegetation index were -0.551 and -0.734 respectively, both passing $p<0.01$ significance test, indicating that the surface temperature had a significant negative correlation relationship with elevation and vegetation index. Therefore, strengthen urban greening is of great significance to the healthy development of the city.

\footnotetext{
* Corresponding author: Juanli Jing, Email: jjlgut2008@163.com
} 


\section{INTRODUCTION}

Land surface temperature (LST) is a significant parameter in exploring the exchange of surface matter, surface energy balance and surface physical and chemical processes (Tomlinson et al., 2011). Human activities have intense impacts on global material cycling and energy flow and gradually become an essential controlling factor in natural ecosystem (Zhou et al., 2016). So, urban thermal environment must be considered in the process of urban development and planning. (Li et al., 2014).

With the opening and reform policy, the Pearl River Delta (PRD) region has experienced rapid urbanization since 1978, which has led to the transition from natural landscapes to impervious surfaces (Song et al., 2014). Therefore, the LST has been changed with the altering of land use/cover. Some scholars have analyzed the LST of PRD region with meteorological station (Zeng et al., 2004; Dou et al., 2010; Cheng et al., 2019). But traditional method based on meteorological stations are difficult to accurately reflect the spatial distribution of LST. With the development of spatial information, remote sensing technology has become one of the important method to retrieval LST. Weng (2001) found the urban development had raised surface radiant temperature by $13.01 \mathrm{~K}$ in the urbanized area by using multi-temporal Landsat Thematic Mapper data and geographic information systems (GIS) technology. Qian et al.(2006) pointed out urban growth had caused LST to raise $4.56^{\circ} \mathrm{C}$ in the newly urbanized part of the PRD region based on Multi-temporal Landsat TM and Landsat ETM+ data. Zhang et al. (2007) found the area of high temperature has increased $250 \mathrm{~km}^{2}$ in the PRD region from 1990 to 2000 based on Landsat TM/ETM+ band 6 images. Wang et al. (2019) analyzed the spatial temporal changes of land cover and the surface urban heat island (SUHI) effect in the Pearl River Delta (PRD) region from 2000 to 2015 by using Landsat and the Moderate Resolution Imaging Spectroradiometer (MODIS), and found the LST increased in general but the spatial pattern of LST increase is affected by the land cover change.

Previous studies in PRD region either mainly focused on certain cities by analyzing meteorological station data or using Landsat data to study the relationship between LST and land use/cover change. And the research region were only include part of the PRD region. There is a lack of comprehensive understanding of LST variation in overall region of PRD. Therefore, this study analyzed the spatial and temporal characteristics of PRD region from 2000 to 2015 based on MODIS data. And fourthly, the correlation between LST and NDVI, elevation had been studied.

\section{STUDY AREA AND DATASETS}

\subsection{Study Area}

The PRD region is located in the Guangdong province, belongs to the southeast China. It consists of nine cities, including Guangzhou, Foshan, Zhaoqing, Shenzhen, Dongguan, Huizhou, Zhuhai, Zhongshan and Jiangmen, and as well as Hong Kong and Macao special administrative regions. This study mainly focused on the nine cities with the longitudes of $112^{\circ}$ to $115.5^{\circ}$ and latitudes of $21.5^{\circ}$ to $24^{\circ}$ (Figure 1). The terrain is high in the north and low in the south, the central area is plain and surrounded by mountains and islands. The PRD region has a subtropical climate. It is warm and humid at four seasons. The average annual temperature is from $21^{\circ} \mathrm{C}$ to $23^{\circ} \mathrm{C}$, whereas the average annual precipitation is more than $1500 \mathrm{~mm}$. From June to October, there are often typhoons. Rainy seasons are coincide with high temperature seasons, and the soil is fertile, rivers are development, which are beneficial to the development of agriculture.

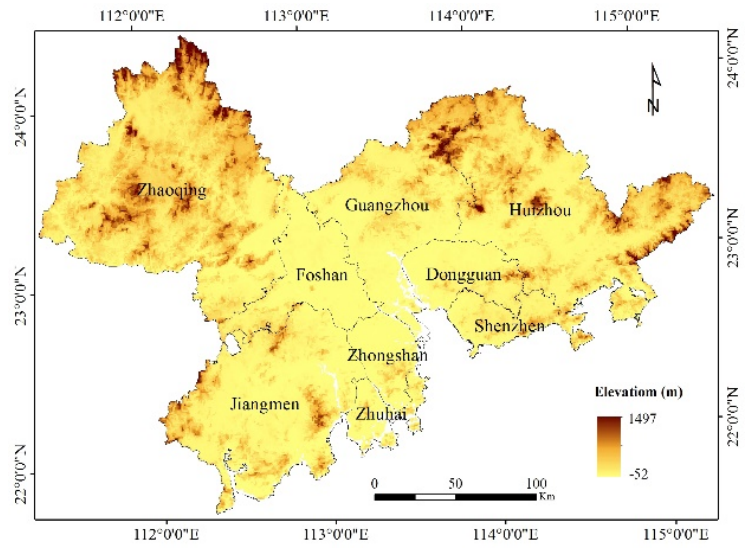

Figure 1. Location of the PRD region

\subsection{Datasets}

The MODIS products were employed in this study due to several advantages. Firstly, it has large spatial coverage which can cover the whole study area. Secondly, it can provide long and continuous time series data and has been used widely. Hence, the MODIS/Terra Monthly global coarse resolution LST data MOD11B3 were selected in this study. The spatial resolution of the selected data was $6 \mathrm{~km}$, and with a sinusoidal projection. The product was synthesized from the daily $6 \mathrm{~km}$ surface temperature/emissivity product (MOD11B1), which is generated by the day/night LST algorithm. The LST data from June to August of 2000, 2006, 2009 and 2015 were used in the study. And it can be downloaded from the National Aeronautics and Space Administration (NASA) site with free of charge (https://ladsweb.modaps.eosdis.nasa.gov/search). In addition, auxiliary data include Digital Elevation Model (DEM) with a resolution of $90 \mathrm{~m}$, which was obtained from Geospatial Data Cloud (http://www.gscloud.cn/). Normalized Differential Vegetation Index (NDVI), land use/cover data and the boundary of the PRD region were obtained from the resource and environmental science data center of Chinese Academy of Sciences (http://www.resdc.cn).

\section{METHOD}

\subsection{The mean standard deviation method}

The mean standard deviation method was used to classify the surface temperature by the combination of the mean and standard deviation of the surface temperature, which can better reflect the classification characteristics of urban heat island than the commonly used equal-spacing density segmentation method (Bai et al., 2013). The standard deviation was a 
reflection of the deviation of surface temperature from the average temperature. Based on the above theories, $\mu$ (mean value), 0.5 std (standard deviation), and 1 std were used as segmentation points to divide the surface thermal field into five levels. This method was adopted to divide the surface thermal field in PRD region, and the classification standard was shown in Table 1. Before the standard deviation method was used, the pixel brightness value should be converted to land surface temperature by the formula $0.02 *$ pixel value-273.15.
In addition, the Pearson correlation coefficient method was utilized to quantitatively analyze the relationship between LST and elevation and NDVI. The correlation between the two variables was represented by $r$, whose value is between -1 and 1. The closer the $r$ value is to 1 , the stronger is the correlation between the two variables.

\begin{tabular}{|c|c|}
\hline Temperature levels & Thermal field partition method \\
\hline High & $T_{S}>\mu+$ std \\
Sub-high & $\mu+0.5$ std $\leq T_{S} \leq \mu+$ std \\
Median & $\mu-0.5$ std $\leq T_{S} \leq \mu+0.5$ std \\
Sub-low & $\mu-$ std $\leq T_{S} \leq \mu-0.5$ std \\
low & $T_{S} \leq \mu-$ std \\
\hline
\end{tabular}

Table 1. The mean standard deviation thermal field partition method

\section{RESULTS AND ANALYSIS}

\subsection{Temporal variation of LST}

The maximum, minimum and mean LST of the PRD region from June to August in 2000, 2006, 2009 and 2015 were compared to analyze the monthly change characteristics of LST (Figure 2). It could be found that the maximum LST was fluctuated from $36.37^{\circ} \mathrm{C}$ to $40.99^{\circ} \mathrm{C}$, appearing in June of 2006 and 2015 respectively. And the maximum LST showed an upward trend from June to August during 2000 to 2015. While the minimum LST was changed significantly in different month, varied from $18.65^{\circ} \mathrm{C}$ to $26.07^{\circ} \mathrm{C}$, and with a slightly rising tendency. The variation of mean LST was not significant.

In further analysis, it could be found that the LST was abnormal in July 2006, the LST was above the mean with $9.69^{\circ} \mathrm{C}$, and the difference between maximum and minimum LST was $19.02^{\circ} \mathrm{C}$, which was higher than the other three years. The reason was that tropical storm No.0604, typhoon No. 0605 and typhoon No.0606 seriously affected Guangdong, which led to frequent high temperatures in July and August.

Moreover, to reveal the variable character of LST in summer (June to August) in 2000, 2006, 2009 and 2015, the LST from June to August in 2000, 2006, 2009 and 2015 were averaged (Figure 3). It could be found that the LST in summer from 2000 to 2015 were relatively high. The maximum LST in summer was increased with year, while the minimum LST was fluctuated and the mean LST was changed gently. The maximum LST was $38.96{ }^{\circ} \mathrm{C}$ and the minimum LST was $25.13{ }^{\circ} \mathrm{C}$ in summer. The difference between maximum and minimum LST in summer from 2000 to 2015 were $11.14^{\circ} \mathrm{C}, 11.39^{\circ} \mathrm{C}, 10.49^{\circ} \mathrm{C}$ and $13.76^{\circ} \mathrm{C}$, respectively.

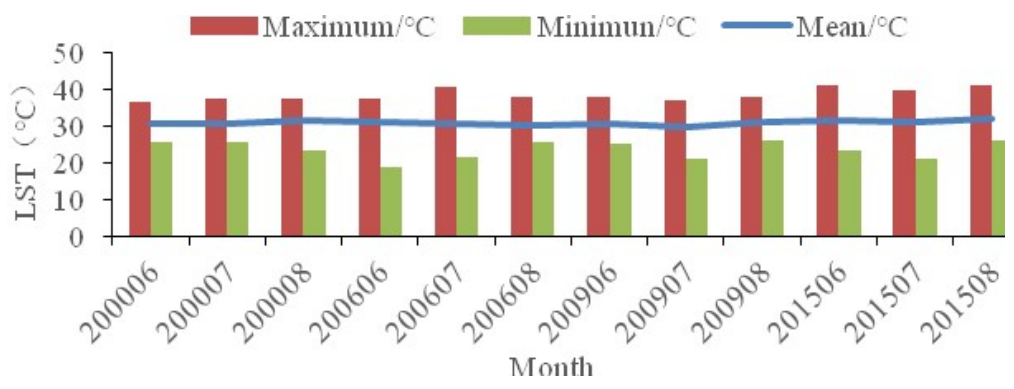

Figure 2. Mean, maximum and minimum LST of June to August from 2000 to 2015 


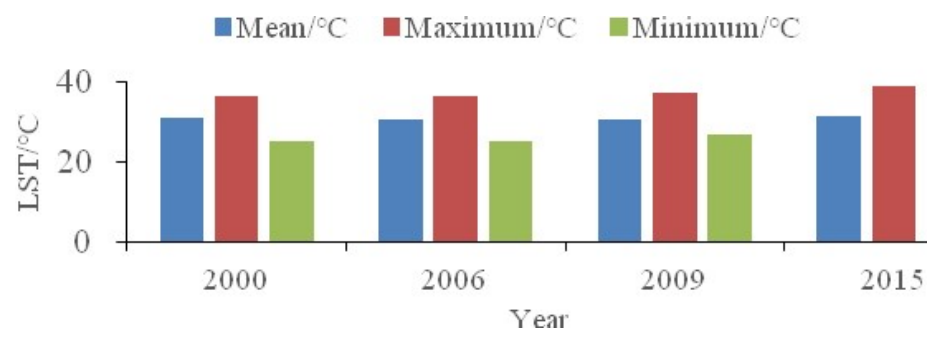

Figure 3. Mean, maximum and minimum LST in summer from 2000 to 2015

\subsection{Spatial pattern of LST}

As shown in Figure 4, the LST in the PRD region from 2000 to 2015 presented a spatial distribution pattern of high in the middle and low around. High LST areas mainly distributed in Guangzhou, Foshan, Dongguan, Shenzhen and other surrounding plain area. While the LST in Zhaoqing, Huizhou and Jiangmen were relatively low. Compared with land use/cover map in PRD region, it could be found that build up areas usually with high LST, but LST were relatively low in areas with good vegetation, and mainly distributed in hilly and mountainous regions.

From the perspective of annual variance, the spatial distribution of LST from June to August were discussed during 2000 to 2015. In the June of 2000 , the areas with LST above $30^{\circ} \mathrm{C}$ were aggregated in the downtown of Foshan, Guangzhou, Dongguan and Shenzhen, and gradual expanded around in July, then covered the entire area of Guangzhou, Dongguan, Shenzhen and Jiangmen in August 2000. However, the spatial distribution of LST above $30^{\circ} \mathrm{C}$ had an opposite changing trend from June to August in 2006, it was mainly occupied in Foshan, Guangzhou, Zhongshan, Dongguan and Shenzhen in June, while shrinked to the downtown of the above cities in August. In the June of 2009 , the distribution of LST above $30^{\circ} \mathrm{C}$ was similar to that of 2006, but it shrinked to the downtown of the distributed cities in July, and then it was spreading around. From June to August in 2015, the spatial distribution of LST above $30^{\circ} \mathrm{C}$ was more wide and intensity in compare with that of 2009, and the high LST areas almost merged into one large area. The spatial distribution characteristics of LST in summer were similar to the monthly variations, the scope of LST was expanding and the intensity was increasing in summer from 2000 to 2015 .

In order to further analysis the spatial pattern of LST in the PRD region. The mean standard deviation of LST from June to August in 2000, 2006, 2009 and 2015 were calculated to reveal the evolution characteristics of LST. The surface thermal field of the study area could be divided into five levels including high, sub high, median, sub low and low. Figure 5 and Table 2 demonstrated the calculation results, the areas of high LST transformed from scatter to aggregation during 2000 to 2015 with the urbanization process. In general, the high LST areas had a fluctuation between months, but there was a similar trend of increasing. Statistic results showed that the percentage of high LST accounted for $14.22 \%, 15.30 \%, 14.78 \%$ and $16.49 \%$ in June from 2000 to 2015 , respectively. And it had a significant increasing trend. The high LST areas were spreading from the downtown of Foshan, Guangzhou, Dongguan and Shenzhen to almost the entire region of above cities and as well as Zhongshan. The percentage of high LST areas in July and August had the same variation trend with June from 2000 to 2015. In addition, the sub high LST areas were dispersed in the PRD region, and had a significant decreasing trend. The median LST areas changed gently during the past 16 years. The sub low LST areas had a tendency of decreasing. To sum up, the high LST areas in PRD region in the summer accounted for $15.07 \%, 14.44 \%, 15.03 \%$ and $15.56 \%$ in 2000 , 2006, 2009 and 2015, respectively. The high LST areas were more concentration in 2015 compared with that of 2000 . Whereas, the low LST areas occupied $15.13 \%, 13.28,12.11$ and $12.33 \%$ from 2000 to 2015 , and with a decreasing tendency. 


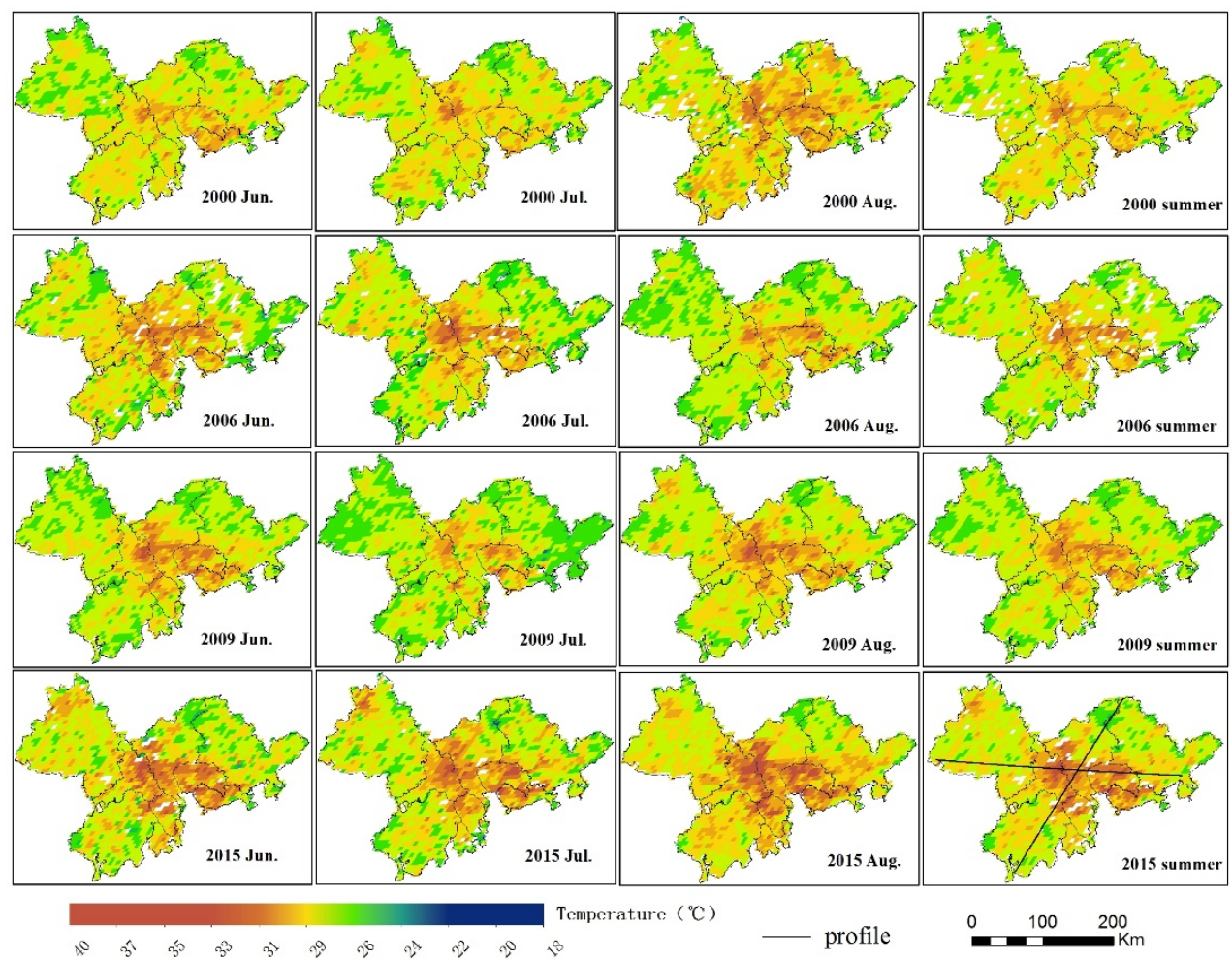

Figure 4. Spatial distribution of LST from June to August of 2000-2015

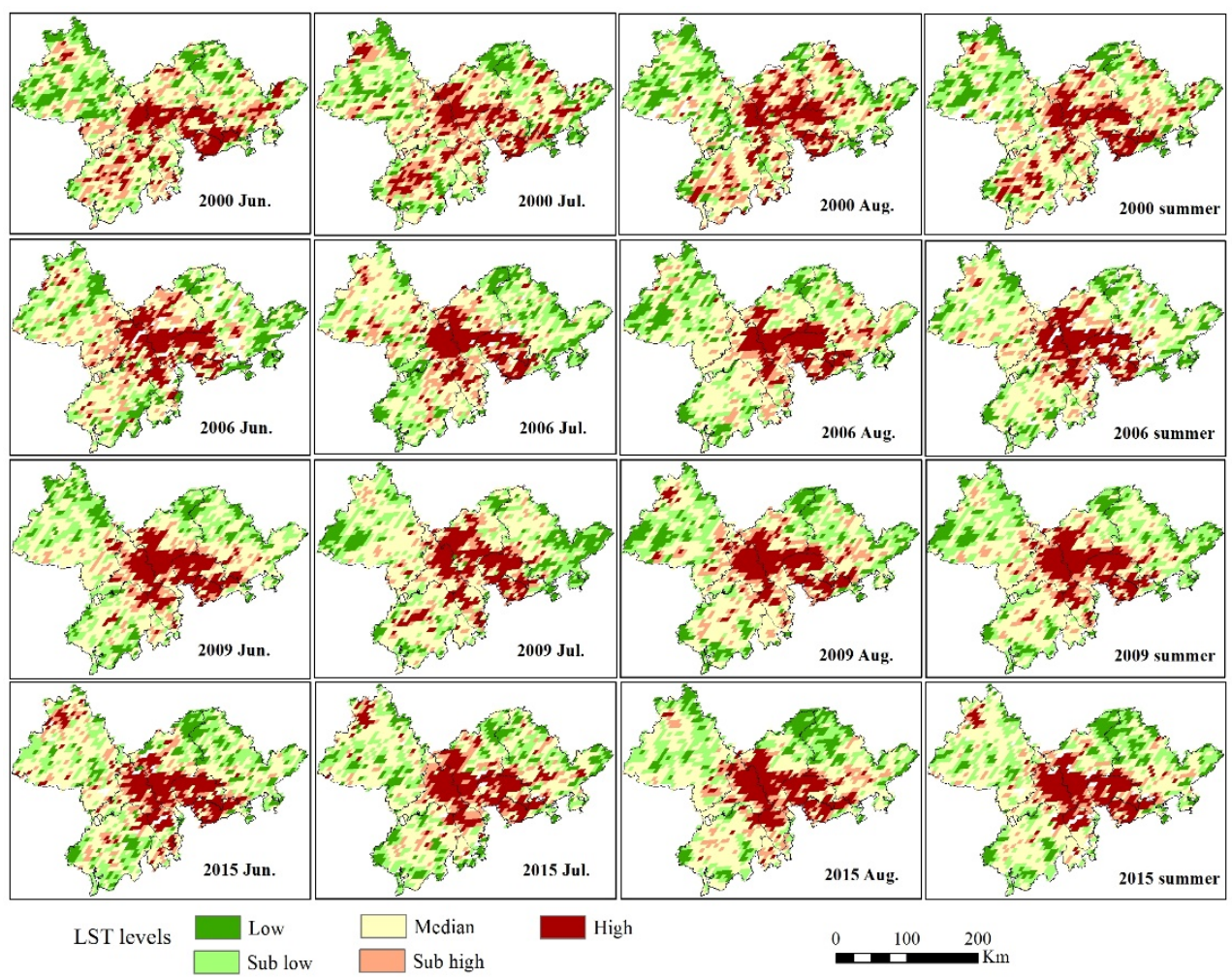

Figure 5. Surface temperature grading map from June to August, 2000-2015

\begin{tabular}{|c|c|c|c|c|c|c|}
\hline \multirow{2}{*}{ Year } & \multirow{2}{*}{ month } & High & Sub High & Median & Sub Low & Low \\
\cline { 3 - 7 } & & \multicolumn{5}{|c|}{ Percentage (\%) } \\
\hline 2000 & 6 & 14.22 & 14.22 & 40.43 & 16.68 & 14.45 \\
& 7 & 15.18 & 15.07 & 38.41 & 16.89 & 14.44 \\
& 8 & 15.71 & 14.35 & 38.04 & 16.83 & 15.06
\end{tabular}




\begin{tabular}{|c|c|c|c|c|c|c|}
\hline & summer & 15.07 & 14.13 & 38.65 & 17.02 & 15.13 \\
\hline \multirow{4}{*}{2006} & 6 & 15.30 & 12.96 & 40.61 & 16.80 & 14.34 \\
\hline & 7 & 14.89 & 11.07 & 41.77 & 19.18 & 13.09 \\
\hline & 8 & 13.36 & 10.79 & 43.80 & 18.90 & 13.14 \\
\hline & summer & 14.44 & 10.12 & 44.18 & 17.98 & 13.28 \\
\hline \multirow{4}{*}{2009} & 6 & 14.78 & 9.36 & 42.24 & 22.32 & 11.30 \\
\hline & 7 & 15.20 & 8.91 & 43.89 & 18.69 & 13.31 \\
\hline & 8 & 14.31 & 11.56 & 36.93 & 18.47 & 18.74 \\
\hline & summer & 15.03 & 9.89 & 39.83 & 23.14 & 12.11 \\
\hline \multirow{4}{*}{2015} & 6 & 16.49 & 10.57 & 39.66 & 20.85 & 12.43 \\
\hline & 7 & 16.40 & 10.74 & 40.42 & 19.11 & 13.34 \\
\hline & 8 & 15.47 & 10.22 & 40.30 & 21.52 & 12.50 \\
\hline & summer & 15.56 & 9.22 & 41.22 & 21.67 & 12.33 \\
\hline
\end{tabular}

Table 2. Statistical analysis of surface temperature in the Pearl River delta region from 2000 to 2015

\subsection{Profile analysis of land surface temperature}

In order to further analyze the spatial variation characteristics of LST in the PRD region, two profiles from west to east and from southwest to northeast were drawn in 2000, 2006, 2009 and 2015 summer, respectively. (Figure 4, Figure 6, Figure 7). The ArcGIS 3D Analyst model was used to create 3D lines based on the LST raster in the selected time.

It could be seen from Figure 6 that the profile from west to east had a bimodal feature, the first peak located in the downtown of Foshan and Guangzhou, and the second peak appeared in the downtown of Dongguan. Before the first peak, the profile was in the scope of Zhaoqing with a relatively high elevation and good vegetation, so the LST was lower than the downtown. After the second peak, there was the city named Huizhou, which had the similar situation to Zhaoqing. Therefore, the LST was relatively low compared with that of downtown. From Figure 7, we could found that the southwest to northeast profile had a character of unimodal. The peak of LST appeared in the downtown of Guangzhou, and the low LST areas mainly distributed in Jiangmen and the north of Guangzhou and Huizhou.

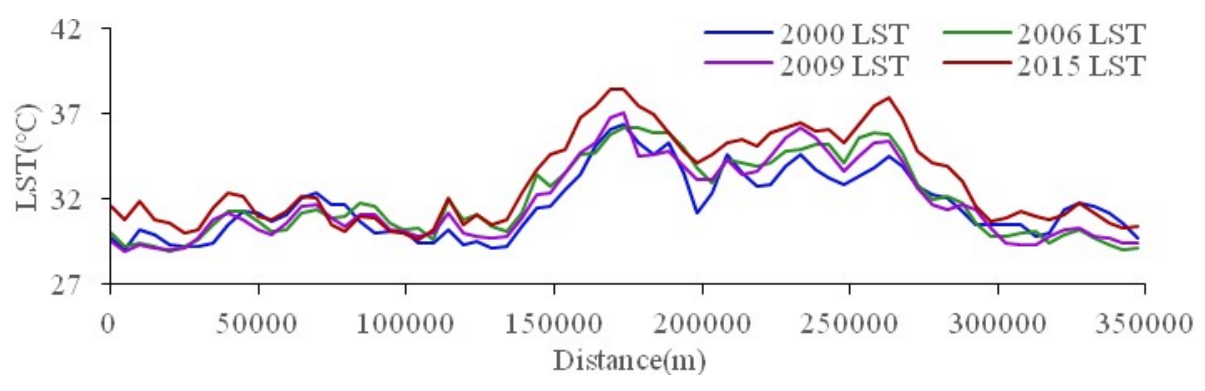

Figure 6. West to East profile in summer from 2000 to 2015

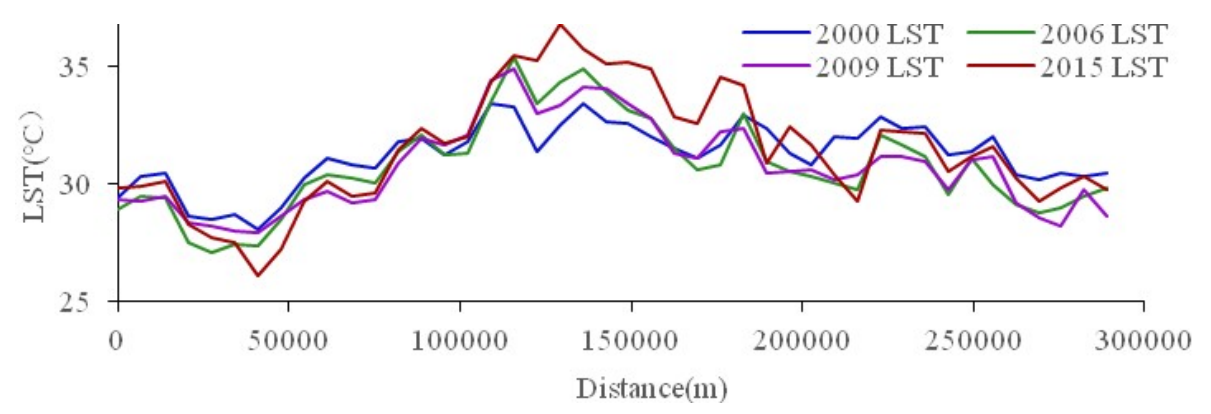

Figure 7. Southwest to Northeast profile in summer from 2000 to 2015

\subsection{Factors affecting surface temperature}

There are lots of factors affecting LST. According to the character of the PRD region, we selected elevation and NDVI as two typical factors to analyze the relationship between LST.
In this study, we selected 2015 summer as an example, the correlation coefficient between LST and elevation, NDVI in the PRD region was researched. Regression statistics were used to analyze the correlation between LST and influenced factors, and scatter plots were made. 
As can be seen from Figure 8, LST and elevation and NDVI were significantly negatively correlated, with correlation coefficients of -0.734 and -0.551 respectively, and both passed the $\mathrm{p}<0.01$ significance test. It indicated that the area with higher altitude and higher NDVI had lower LST, while the area with lower altitude and lower NDVI had relatively higher LST. In terms of land use types, the LST in residential and industrial areas were relatively high, while that of vegetation-covered areas was relatively low.
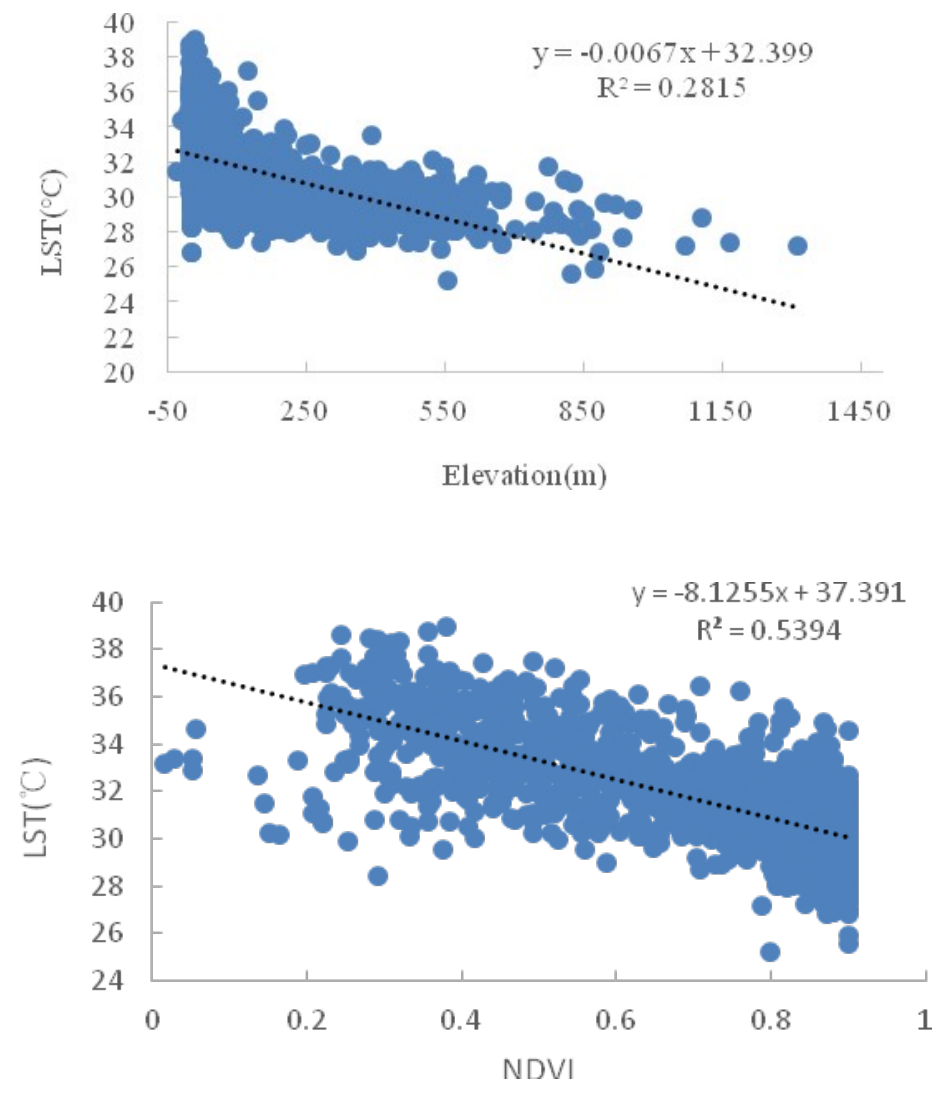

Figure 8. Scatter plot of LST and elevation and NDVI of summer in 2015

\section{CONCLUSIONS}

The following conclusions were drawn in this study:

(1) From 2000 to 2015, the LST in the PRD region showed an upward trend in summer, with a spatial distribution pattern of high in the middle and low around. With the development of urbanization, the high temperature area evolved from dispersion to concentration, and showed the trend of spreading around.

(2) Surface temperature profile analysis showed that the LST in built-up areas were relatively high, while that in suburban areas were relatively low.

(3) Correlation analysis demonstrated that the correlation between LST and elevation and NDVI were significantly negative. High temperature areas were mainly distributed in built-up areas with relatively low altitude and sparse vegetation. While, the low-temperature areas were mostly in mountainous and hilly areas with relatively high altitude and dense vegetation.

(4) In this study, the spatial resolution of monthly MOD11B3 LST dataset is $6 \mathrm{~km}$. which may influence the accuracy of the research results. So the following studies should focus on the use of high-resolution satellite data to improve the accuracy of LST inversion. In addition, factors influencing LST are many and complex, we should combined with multi-factors for further comprehensive analysis.

\section{ACKNOWLEDGEMENTS}

This research was financially supported by Guangxi Key Laboratory of Spatial Information and Geomatics (16-380-25$08)$, Guangxi young and middle-aged teacher's basic ability improvement project (KY2016YB192).

\section{REFERENCES}

Bai, Y., Meng, H., Wang, M., Su, J.H., Sha, C.Y., Ruan, J.J., 2013. Spatial and temporal changes of urban thermal landscape pattern in Shanghai. Environmental Science \& Technology, 36 (3), 96-201. (in Chinese)

Cheng, D., Wang, Y.W., Liu, S.D., Xiao, F., 2019. Spatial and temporal distribution characteristic of summer heat waves in the Pearl River delta region during 1959-2012 and analysis of the impact of urban heat island effect. Science Technology and Engineering, 19 (1), 273-283. (in Chinese) 
Dou, H.Y., Zhang, J.J., Zhao, X.Y., 2010. Study on spatial distribution and intensity of urban heat island in Pearl River Delta. Areal Research and Development, 29 (4), 72-77. (in Chinese)

Li, X.Z., Li, H.Y., Zhang, Q.T., Qiu, G.Y., 2014. Study on reducing effect of different urban landscapes on urban temperature. Ecology and Environmental Sciences, 23(1), 106112. (in Chinese)

Qian, L.X., Cui, H.S., Chang, J., 2006. Impacts of Land Use and Cover Change on Land Surface Temperature in the Zhujiang Delta. Soil Science Society of China, 16(6), 681-689.

Song, J., Du, S.H., Feng, X., Guo, L., 2014. The relationships between landscape compositions and land surface temperature: quantifying their resolution sensitivity with spatial regression models. Landsc. Urban Plan, 123, 145-157.

Tomlinson, C.J., Chapman, L., Thrones, J.E., Baker, C., 2011. Remote sensing land surface temperature for meteorology and climatology: a review. Meteorological Applications, 18, 296306.
Weng, Q. 2001. A remote sensing-GIS evaluation of urban expansion and its impact on surface temperature in the Zhujiang Delta, China. International Journal of Remote Sensing, 22(10), 1999-2014.

Wang, R., Cai, M., Ren, C., Bechtel, B., Xu, Y., Ng, E., 2019. Detecting multi-temporal land cover change and land surface temperature in Pearl River Delta by adopting local climate zone. Urban Climate, https://doi.org/10.1016/j.uclim.2019.100455

Zhou, D.C., Zhang, L.X., Hao, L., Sun, G., Liu, Y.Q., Zhu, C., 2016. Spatiotemporal trends of urban heat island effect along the urban development intensity gradient in China. Science of the Total Environment, 544, 617-626.

Zeng, X., Qian, G.M., Pan, W.J., 2004. Study on urban heat island effect in Pearl River Delta urban group. Meteorological Monthly, 30 (10), 12-16. (in Chinese)

Zhang, J., Wang, Y., Wang, Z., 2007. Change analysis of land surface temperature based on robust statistics in the estuarine area of Pearl River (China) from 1990 to 2000 by Landsat TM/ETM+ data. International Journal of Remote Sensing, 28 (10), 2383-2390. 